\title{
CAPÍTULO 46
}

\section{CARACTERIZAÇÃ̃o DO MODELO DE PLANEJAMENTO E OPERAÇÃO DE REATORES NUCLEARES DE ENSINO, PESQUISA \& PRODUÇÃO: APLICAÇÃO AO IEA-R1 IPEN-CNEN/SP}

Aline A. Perini, Cassiane R. Jaroszewski, Anderson Z. Freitas

Instituto de Pesquisas Energéticas e Nucleares - IPEN-CNEN/SP

Av. Professor Lineu Prestes, 2242

05508-000 São Paulo - SP

aline.perini@ipen.br

\section{RESUMO}

No Este estudo piloto é resultado de um projeto de doutorado em colaboração com CRPq (Centro do Reator de Pesquisas) e o NIT IPEN-CNEN/SP (Núcleo de Inovação Tecnológica do Instituto de Pesquisa Energéticas e Nucleares da Comissão Nacional de Energia Nuclear) sob o campo do conhecimento da qualidade dos indicadores de C,T\&I (Ciência, Tecnologia \& Inovação). Instituições de alto nível de governança, reforçam e legitimam a função de desenvolvimento e inovação ante as 
transformações científicas, tecnológicas, econômicas, sociais, históricas, políticas, geográficas, ambientais, produtivas ao longo do tempo. Coloca como cerne o papel da gestão ao mapear fenômenos da formação da identidade histórica ao passo que pavimenta os caminhos para a construção do futuro mais estrutural, alçado em tecnologias emergentes para soluções de problemas de cidades inteligentes. As tecnologias nucleares, de uso pacífico, apresentam propriedades de convergência e habilitadoras nos mais diversos pontos de sistemas produtivos. Utilizando-se informações divulgadas no Plano Diretor da AIEA (Agência Internacional de Energia Nuclear) e do IPEN de 2012 a 2018 aplicou-se metodologia DEA associada aos componentes principais da Tripla Hélice Híbrida, respaldada no equilíbrio das relações. Como resultado desta iniciativa, foram constatadas sinergias totais, evidenciando o fluxo da interface das relações entre Governo, Universidade e Indústria, propondo de forma inédita a aplicação metodológica conjunta DEA Tripla Hélice ao CRPq para regular transições entre passado e futuro das Instituições de Ciência e Tecnologia para países em desenvolvimento.

\section{INTRODUÇ̃̃OO}

Estudos do futuro têm sido referenciados na literatura como desafio estratégico em antecipar tendências e preparar as instituições de forma a aumentar a taxa de sobrevida e permanecer ao longo do tempo minimizando efeitos colaterais sistêmicos e, exposição à vulnerabilidade do ambiente $[1,5,10]$.

Nesta perspectiva, ao longo dos desdobramentos socioeconômicos foram propiciados diversos mecanismos administrativos, legais, econômicos, sociais, políticos e institucionais consistindo na trajetória para alavancar a economia de países em desenvolvimento na criação de sinergias, parcerias, redes e colaborações $[2,3,4,9]$.

Diversas são as tecnologias convergentes e habilitadoras, ou tecnologias embarcadas no modelo chave-fechadura advindas das técnicas nucleares pacíficas, haja visto o marco de fundação do IEA-R1 e a trajetória institucional do IPENCNEN/SP protagonizando a oferta de soluções ao campo da saúde, especialmente ao diagnóstico e tratamento de câncer, reforçando e legitimando sua importância ao desenvolvimento social regional, nacional e internacional [7] neste campo científico.

Embora este estudo aponte interesse por estudos do futuro [1], preconizou de forma inédita a análise DEA (Análise Envoltória de Dados) [2,3,4] associada aos componentes principais G-U-I (Governo-Universidade-Indústria) do conjunto Tripla Hélice [4], relacionando os fatores produtivos e sinérgicos do CRPq. Foram 
delineadas e caracterizadas as melhores práticas, colocadas à disposição de gestores públicos, desenvolvedores de políticas públicas, cientistas, empreendedores, estudantes e demais interessados.

A capacidade de planejar e compreender fenômenos do passado constitui-se um ferramental poderoso na criação de sinergia em ambientes dinâmicos na era do conhecimento [9]. Remarca a importância da visão estratégica da administração para a regular transição entre a identidade histórico-cultural e a complexidade da sociedade em seu entorno para o século XXI [1,4,9].

\section{METODOLOGIA E RESULTADOS}

O objetivo deste estudo piloto foi caracterizar o Centro do Reator Nuclear de Pesquisas (CRPq) de uma ICT (Instituição de Ciência e Tecnologia) do IPENCNEN/SP USP de forma a validar a aplicação conjunta dos modelos encontrados na literatura científica como mais bem estabelecida em momentum de transformações para países em desenvolvimento alinhada às políticas de cooperação para o desenvolvimento da AIEA (Agência Atômica Internacional) [6].

Por meio de método DEA comparativo longitudinal de dados disponíveis no Plano Diretor IPEN de 2012 a 2018, aplicou-se uma proxy de componentes principais G-U-I (Governo-Universidade-Indústria). Foram catalogadas as entradas (In) e saídas (out) de um sistema produtivo para diagnóstico da função expressa.

Os dados coletados manualmente do Plano Diretor IPEN foram inseridos em planilha eletrônica Excel ${ }^{\circledR}$ e auditados após consolidação. Suplementos para análise de dados e otimização da função foram aplicados. Realizou-se plotagem de gráfico de dispersão com o melhor cenário, expressando a função linear apresentada ao sistema produtivo do $\mathrm{CRPq}$, bem como seu coeficiente de Determinação $\mathrm{R}^{2} \mathrm{e}$ coeficiente de correlação R.

Na plotagem, foi demarcada a fronteira de eficiência do sistema produtivo, envolvendo todo o conjunto de dados obtidos pela função DEA.

Para a classificação das proxys, foram realizadas análises do sistema mercadoria da teoria de recursos, de acordo com a literatura econômica contábil da economia do conhecimento e intangibilidade [1]. Quatro cenários foram construídos, valendo-se de entrada de dados no ano de 2012 apresentado resultados no mesmo ano em 2012 $(\mathrm{t}=0)$. Os demais cenários foram elaborados considerando as entradas do sistema de 2012 com saídas do sistema nos anos de 2013 ou 2014 ou 2015, ou seja, $t+1$; $\mathrm{t}+2, \mathrm{t}+3$ respectivamente. A melhor correlação e simplificação da função linear foi apresentada no $\mathrm{t}=0$. Os demais resultados foram descartados. 
Por fim, foram compiladas tabelas para indicação do fluxo aos componentes principais G-U-I e cálculo dos índices de sinergia encontrados.

\subsection{Resultados e Análise Envoltória de Dados}

De acordo com dados do plano diretor da AIEA (Agência Internacional de Energia Atômica) [6], gráfico 1 foram mapeadas as áreas temáticas prioritárias dos biênios (2015- 2016) e (2018-2019) para cooperação e desenvolvimento de uso pacífico da tecnologia nuclear.

Figura 1 - Mapa de áreas temáticas prioritárias para cooperação e desenvolvimento da agência atômica internacional.

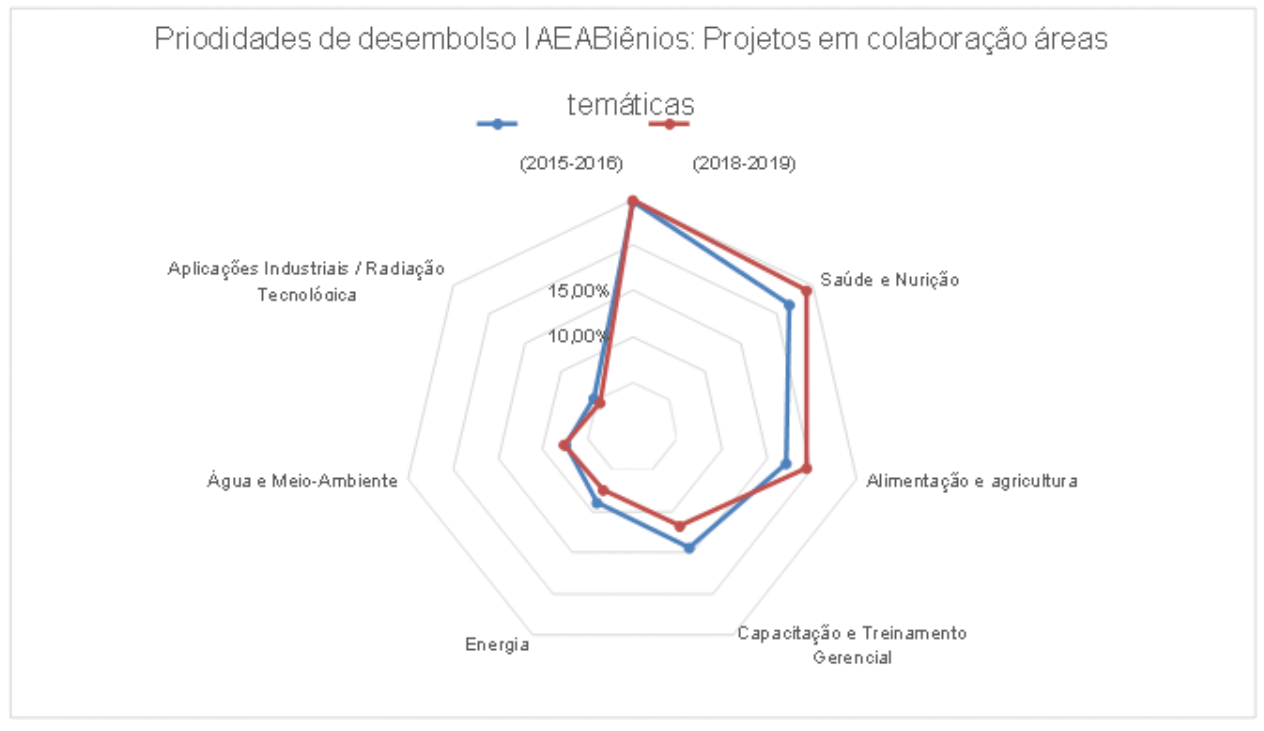

Fonte: Elaborado pelos autores.

Utilizando dados do Plano Diretor CRPq IPEN-CNEN/SP de 2012 a 2018 aplicadado a metodologia DEA foi gerado o Gráfico 2. A imagem da função $F(x)$ é representada pelo eixo "y", denotando os resultados consolidados (out) ou saídas do sistema produtivo. $\mathrm{O}$ eixo " $\mathrm{x}$ " é representado pelas entradas consolidadas do sistema produtivo. 
Figura 2 - DEA aplicado ao CRPq IEA-R1.

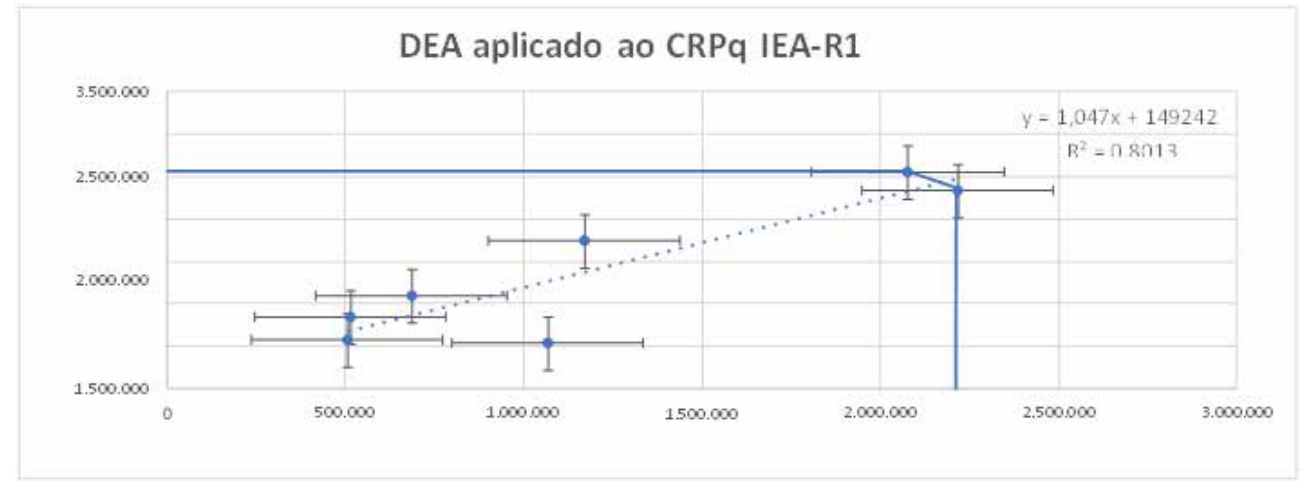

Fonte: Elaborado pelos autores.

$\mathrm{O}$ cenário $\mathrm{t}=0$ apresentou o coeficiente de determinação linear $\mathrm{R}^{2}=0,8013 \mathrm{e}$ correlação $=0,8951$. Ou seja, as entradas e saídas do Sistema produtivo do CRPq de 2012 a 2018 são computados dentro do mesmo ano. A relação entre entradas e saídas do sistema é explicada em $80,13 \%$ pelo algotitmo (1):

$\mathrm{y}=1,047 \mathrm{x}+149.242$

O Plano Diretor para cumprir com a função de planejamento, pode-se utilizar desta ferramenta para a construção de cenários futuros, conferindo linearidade temporal objetiva das decisões estratégicas do CRPq para construção de cenários futuros.

\subsubsection{Aplicação componentes principais: DEA, Tripla Hélice e Sinergia}

Dada a reta de regressão linear que estabelece a relação de equilíbrio entre os pontos o gráfico 1, ou seja, onde estabelece congruência de área, a medida de sinergia total foi calculada como o resultado das saídas(s) eixo (y, out) menos as entradas(s) eixo (x, in). A diferença entre produtividade e eficiência é que a eficiência expressa a relação ótima entre recursos consumidos e resultados gerados, enquanto a sinergia do sistema é dada pela diferença total.

A Tabela 1 distribui os componentes da Tripla Hélice G-U-I (GovernoUniversidade- Indústria) como protagonistas do conjunto do sistema e posiciona a Universidade como maior geradora de sinergia, seguida pela indústria, enquanto o Governo é caracterizado como mantenedor deste sistema positivo, realizando o papel ancoragem, mantenedor e fomentador. Estas descobertas aplicadas no 
CRPq corroboram a teoria da literatura científica Tripla Hélice, otimizado pela aplicação DEA de um sistema produtivo.

Tabela 1 - Componentes principais DEA Tripla Hélice do CRPq

\begin{tabular}{|c|c|c|c|c|}
\hline DEA Tripla Hélice & out & in & Total Geral & Sinergia \\
\hline G & 3.495 .672 & 6.402 .181 & 9.897 .853 & -2.906 .509 \\
\hline U & 5.753 .838 & 476 & 5.754 .314 & 5.753 .362 \\
\hline I & 2.247 .211 & 159 & 2.247 .370 & 2.247 .052 \\
\hline Total Geral & 11.496 .720 & 6.402 .816 & 17.899 .536 & 5.093 .905 \\
\hline
\end{tabular}

A Tabela 2 demonstra a matriz da relação DEA aos componentes principais Tripla Hélice em relação ao total geral consolidado. É possível verificar que o governo se caracteriza principalmente como provedor chave, fomentador de recursos ao sistema produtivo, enquanto a indústria e a universidade surgem como hélices fechaduras, configurando uma sinergia total do sistema CRPq DEA Hélice(s) de $28 \%$.

Tabela 2 - Sinergia CRPq DEA Hélice(s)

\begin{tabular}{|c|c|c|c|c|}
\hline DEA Tripla Hélice & out & in & Total Geral & Sinergia \\
\hline G & $35 \%$ & $65 \%$ & $100 \%$ & $-29 \%$ \\
\hline U & $100 \%$ & $0 \%$ & $100 \%$ & $100 \%$ \\
\hline I & $100 \%$ & $0 \%$ & $100 \%$ & $100 \%$ \\
\hline Total Geral & $64 \%$ & $36 \%$ & $100 \%$ & $28 \%$ \\
\hline
\end{tabular}

A Tabela 3 demonstra índices da análise vertical, indicando a distribuição do sistema sinérgico até a sexta hélice. Verifica-se que a relação da interface CRPqUniversidade é um componente-chave de sinergia do sistema para o surgimento de novas hélices, sendo responsável em $50 \%$ dos resultados gerados totais.

Tabela 3 - Análise vertical CRPq DEA Hélice(s)

\begin{tabular}{|c|c|c|c|c|}
\hline DEA Tripla Hélice & out & in & Total Geral & Sinergia \\
\hline G & $30 \%$ & $100 \%$ & $55 \%$ & $-57 \%$ \\
\hline U & $50 \%$ & $0 \%$ & $32 \%$ & $113 \%$ \\
\hline I & $20 \%$ & $0 \%$ & $13 \%$ & $44 \%$ \\
\hline Total Geral & $100 \%$ & $100 \%$ & $100 \%$ & $100 \%$ \\
\hline
\end{tabular}




\section{CONCLUSÕES}

As áreas temáticas de prioridade de uso pacífico da tecnologia nuclear para cooperação e desenvolvimento são: segurança (25\%), saúde e nutrição (24\%), alimentação e agricultura (20\%) e capacitação e treinamento gerencial (15\%). Energia, água e meio-ambiente e aplicações industriais completam o restante (16\%).

O modelo de interação DEA Tripla Hélice Híbrido é adequado a realidade do CRPq; foi constatado que a sinergia total do arranjo de colaboração G-U-I CRPq IPEN-CNEN/SP foi de $28 \%$. A sinergia é explicada principalmente pelo fluxo de interação do Governo, posicionado como chave-mestra, tendo a Universidade e a Indústria como receptoras fechaduras para do desenvolvimento das competências precisas na economia do conhecimento do século XXI.

\section{AGRADECIMENTOS}

Agradecimentos à Capes e à administração do IPEN-CNEN-SP por propiciar a realização deste estudo.

\section{REFERÊNCIAS}

1. PERINI,. A. A. Clientes como ativos patrimoniais: práticas e capacidades gerenciais. [S.l.]: Novas Edições Acadêmicas, 2019.164 p. ISBN 978-613-976428-0.

2. CHARNES, A.; COOPER, W. W.; RHODES, E. Measuring the efficiency of decision making units. European Journal of Operational Research, v. 2, n. 6, p. 429-444, 1978. Disponível em: https://doi.org/10.1016/0377-2217(78)90138-8.

3. COOPER, W.W.; SEIFORD, L. M.; TONE, K. Data envelopment analysis: a comprehensive text with models, applications, references and DEA-Solver software. Dordrecht/Holanda: Kluwer Academic Publishers, 1999. 352 p.

4. ETZKOWITZ, H.; ZHOU, C. Hélice Tríplice: inovação e empreendedorismo universidade-indústria-governo. Estudos Avançados, v. 31, n. 90, 2017. Disponível em: http://dx.doi.org/10.1590/s0103-40142017.3190003.

5. FARRELL, M. J. The measurement of productive efficiency. Journal of the Royal Statistical Society: series A, v. 120, n. 3, p. 253-290, 1957. DOI: 10.2307/2343100 Disponível em: https://www.jstor.org/stable/2343100.

6. INTERNATIONAL ATOMIC ENERGY AGENCY. IAEA Annual Report 2017. Management of technical cooperation for development. Disponível em: 
https://www.iaea.org/sites/default/files/publications/reports/2017/gc62-3-tc.pdf. Acesso em: 2019.

7. IPEN. Instituto de Pesquisa Energéticas Nucleares. Disponível em: https:// www.ipen.br/portal_por/portal/default.php. Acesso em: 2019.

8. SEllitTO, M. A.; RIBEIRO, J. L. D. Construção de indicadores para avaliação de conceitos intangíveis em sistemas produtivos. Gestão \& Produção, v. 11, n. 1, p. 75-90, jan.-abr. 2004. Disponível em: http://dx.doi.org/10.1590/ S0104-530X2004000100007.

9. WANG, C.; XIE, F. Corporate governance transfer and synergistic gains from mergers and acquisitions. The Review of Financial Studies, v. 22, n. 2, p. 829858, 2009. Disponível em: https://doi.org/10.1093/rfs/hhn018.

10. PERINI, A. A.; OUTAMHA, R. IMPRESSIONS of II International Triple Helix Summit Dubai, United Arab Emirates - November 2018. Hélice Magazine, v. 7, n. 4, p. 6-9, 2018. Disponível em: https://www.triplehelixassociation.org/ helice/volume-7-2018/helice-issue-7- 4/impressions-of-ii-international-triplehelix-summit-dubai-united-arab-emirates- november-2018. 